\title{
EVALUACIÓN DEL ESTADO TRÓFICO Y COMPARACIÓN DE MODELOS RELATIVOS AL FÓSFORO EN LOS EMBALSES DE CERNADILLA Y VALPARAÍSO (RÍO TERA, ZAMORA)
}

\author{
H. Fraile, E. Orive y J. Pozo \\ Lab. Ecología, F. Ciencias, Universidad del País Vasco /E.H.U., Apdo. 644,48080 Bilbao, España.
}

Palabras clave: Embalses, Estado trófico, Carga crítica de fósforo.

Key words: Reservoirs, Trophic State, Critical phosphorus load.

\begin{abstract}
TROPHIC STATE EVALUATION AND COMPARISON OF MODELS RELATED TO PHOSPHORUS IN CERNADILLA AND VALPARAISO RESERVOIRS (TERA RIVER, ZAMORA)

Cernadilla (1965) and Valparaíso (1988) reservoirs, located in the river Tera which is a tributary within the Duero basin (Zamora), are used to produce electric energy and provide irrigation. The trophic state of the headwater reservoir (Cernadilla) has became worse since Valparaíso construction. In 1991, the internal phosphorus loading was 120,97 mg TP.m² summer in Cernadilla, and 30,92 mg TP. $\mathrm{m}^{2}$ summer in Valparaíso. These estimates were calculated from the increased mass of hypolimnetic phosphorus divided by the surface area of the reservoir. The rates of phosphorus release from anoxic sediment surfaces were 14,40 and 6,2 mg TP.m.2 $\mathrm{d}^{1}$, in Cernadilla and Valparaíso respectively. Both reservoirs were classified as mesotrophic in 1991.
\end{abstract}

\section{INTRODUCCIÓN}

En las últimas décadas se han construído más de 1.000 embalses en el Territorio de la Península Ibérica, lo que nos sitúa a la cabeza de Europa en cuanto a la regulación de caudales. A pesar de ese gran número de embalses, la mayor parte de los estudios de este tipo de sistemas en España se ha realizado en los últimos 20 años. El más significativo, entre los trabajos pioneros, es el de MARGALEF et al., (1976), que permitió la caracterización físico-química y biológica de 104 embalses y que ha sido recientemente actualizado (RIERA et al., 1991; 1992). En la cuenca del río Tera se han realizado varios estudios ambientales. Algunos hacen referencia al Lago de Sanabria (VEGA et al., 1992), mientras que otros se centran más en la problemática creada por los embalses de Cernadilla y Valparaíso (LIMNOS S.A., 1989; BENGOECHEA, 1991; FRAILE, 1994; POZO et al., en prensa). La eutrofización es el principal problema que se plantea en las aguas represadas, siendo el fósforo, el nutriente clave para su control. Este trabajo pretende reflejar el estado trófico de los embalses de Cernadilla y Valparaíso durante el año 1991 y discute la aplicación de diversos modelos empíricos relativos al fósforo que hacen referencia a la calidad del agua embalsada y a su posible evolución trófica.

\section{ÁREA DE ESTUDIO}

El sistema de embalses en cadena formado por Cernadilla (1965), Valparaíso (1988) y más recientemente, Nuestra Sra de Agavanzal (1994), se encuentra en el tramo medio-alto del río Tera, subafluente del Duero, en la provincia de Zamora (Figura 1). Estos embalses son utilizados por IBERDROLA S.A. para producir energía eléctrica de puntas durante el período crítico (Noviembre-Febrero), turbinando para obtener energía extra en situaciones de mayor demanda energética. Sin embargo, el sistema cuenta con la servidumbre de un suministro de agua para riego en verano, garantizando en todo caso un caudal mínimo de $3 \mathrm{~m}$ ' seg ' Según las operaciones realizadas en los embalses en 1991, Cernadilla es el embalse que más fluctúa, alcanzando su nivel máximo en Mayo y perdiendo cerca de $200 \mathrm{Hm}$ ' durante el verano. Por el contrario, Valparaíso sufre fluctuaciones inferiores a $3 \mathrm{~m}$ de su nivel máximo normal (FRAILE, 1994). La toma de agua para turbina se encuentra a 20 y $15 \mathrm{~m}$ del 
TABLA 1. Principales parámetros morfométricos e hidrológicos de los embalses de Cernadilla y Valparaíso.

TABLE 1. Main rnorphonietrical and hydrologienl features of Cernadilla and Valparaíso reservoirs.

\begin{tabular}{|c|c|c|}
\hline EMBALSE & Cernadilla & Valparaíso \\
\hline Volumen máximo & $220,87 \mathrm{Hm}^{3}$ & $163,20 \mathrm{Hm}^{3}$ \\
\hline Volumen útil & $198.57 \mathrm{Hm}^{3}$ & $103,20 \mathrm{Hm}^{3}$ \\
\hline Cota normal máxima & $889,00 \mathrm{~m}$ & $833,00 \mathrm{~m}$ \\
\hline Nivel mínimo de explotación & $856,00 \mathrm{~m}$ & ---- \\
\hline Area superficial máxima(A) & $1189,70 \mathrm{Ha}$. & $1216,50 \mathrm{Ha}$. \\
\hline Profundidad máxima (Zm) & $54,00 \mathrm{~m}$ & $52,00 \mathrm{~m}$ \\
\hline Profundidad media (z) & $18,56 \mathrm{~m}$ & $13,40 \mathrm{~m}$ \\
\hline Profundidad relativa $(\mathbf{Z r})$ & $1,38 \%$ & $1,32 \%$ \\
\hline Longitud máxima & $21,51 \mathrm{Km}$ & $22,04 \mathrm{Km}$ \\
\hline Anchura máxima & $0,95 \mathrm{Km}$ & $1,69 \mathrm{Km}$ \\
\hline Perímetro & $85,07 \mathrm{Km}$ & $88,21 \mathrm{Km}$ \\
\hline Desarrollodel perímetro & 6,95 & 7,13 \\
\hline Desarrollo del volumen (Dv) & 1,03 & 0,77 \\
\hline Cocientez:Zm & 0,34 & 0,25 \\
\hline \multicolumn{3}{|l|}{ CUENCA } \\
\hline $\begin{array}{l}\text { Superficie de la cuenca vertiente } \\
\text { Tipo de cuenca } \\
\% \text { cuenca ocupado por el embalse }\end{array}$ & $\begin{array}{l}576,00 \mathrm{Km}^{2} \\
\text { silícea } \\
2,06 \%\end{array}$ & $\begin{array}{l}798,00 \mathrm{Km}^{2} \\
\text { silícea } \\
1,52 \%\end{array}$ \\
\hline \multicolumn{3}{|l|}{ ANO 1991} \\
\hline Volumen medio ( $\mathrm{Hm}^{3}$ ) & 119,323 & 151,38 \\
\hline Cota media (m) & 875,23 & 832,03 \\
\hline Entradas $\left(\mathrm{Hm}^{3}\right)$ & 202,30 & 303,82 \\
\hline Salidas $\left(\mathrm{Hm}^{3}\right)$ & 294,96 & 298,94 \\
\hline Superficie media (Ha) & 640,10 & 1216,50 \\
\hline Profundidad media (m) & 18,65 & 12,44 \\
\hline \multicolumn{3}{|l|}{ Tiempo de retención hidráulico } \\
\hline (Tw: años) & 0,40 & 0,50 \\
\hline Tasa de renovación (D: años -1) & 2,47 & 1,97 \\
\hline Carga hidraúlica ( $\mathrm{q}_{\mathrm{s}}: \mathrm{m} /$ años -1$)$ & 46,62 & 24,88 \\
\hline
\end{tabular}

fondo en Cernadilla y Valparaíso, respectivamente y la de desagüe de fondo a 5 y $3 \mathrm{~m}$, respectivamente. Los principales parámetros morfométricos e hidrológicos tanto a nivel general, como a nivel particular para el año 1991, aparecen en la Tabla 1.

Se encuentran en un área poco humanizada $\left(9\right.$ hab. $\left.\mathrm{Km}^{-2}\right)$ y presentan agua de mincralización baja, como corresponde al sustrato silíceo de su cuenca. Pertenecen según la clasificación regional de MARGALEF et al. (1976) al grupo I, que corresponde a los embalses de la zona Oeste de la Península, con poco calcio y azufre, que condiciona una mayor tendencia hacia la eutrofización si la carga de fósforo es excesiva (RIERA et al. 1992; LÓPEZ \& MORGUI, 1993). 


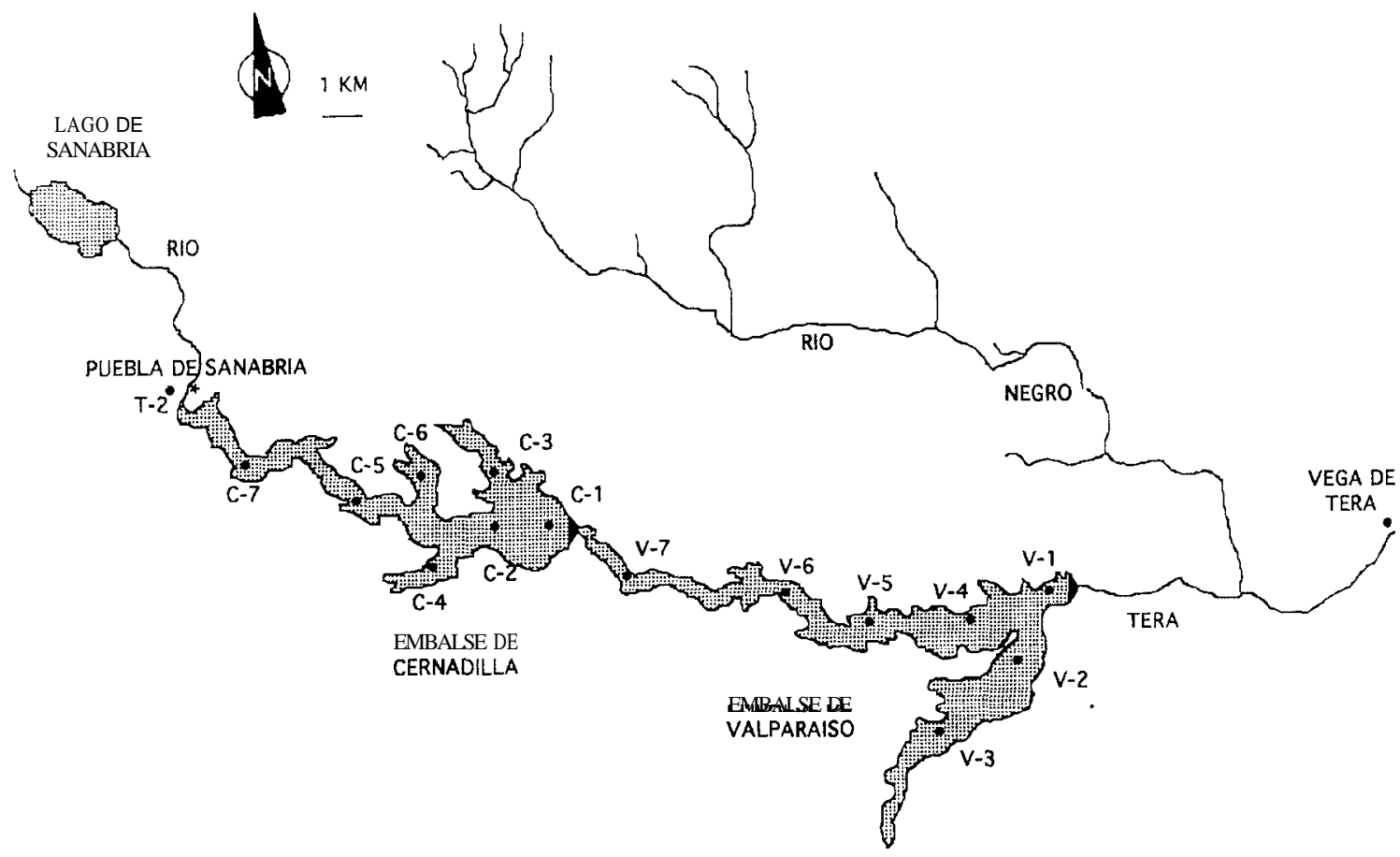

FIGURA 1. Área de estudio. Situación de las estaciones de muestreo en el cauce fluvialy en los embalses de Cernadilla y Valparaíso.

FIGURE 1. Study site. Regulation of the river Tera by the Cernadilla and Valparaíso reservoirs. The location of sampling stations and main villages are indicated.

\section{MATERIAL Y MÉTODOS}

\section{Estrategia de muestreo}

Se tomaron muestras quincenalmente desde Mayo de 1991 hasta Abril de 1992 en una estación situada antes de los embalses (est. T-2) y en una estación cercana a la presa en ambos casos (est. C-1 y V-1). Durante el período de estratificación térmica, esto es, de Mayo a Octubre de 1991, se realizaron 5 campañas intensivas de muestreo que abarcaron el eje longitudinal de los embalses, así como sus principales brazos laterales. Se situaron 7 estaciones de muestreo (Figura 1), recogiéndose agua a 3, 4 ó 5 profundidades, en función del espesor de la columna de agua. Las determinaciones llevadas a cabo in situ mediante sensores de campo fueron las siguientes: temperatura y oxígeno (oxímetro WTW, OXI 196), pH (pHmetro WTW, PH 196) y conductividad (conductivímetro WTW, LF 191). También se midió la transparencia del agua a partir de la profundidad de visión del disco de Secchi y la clorofila 'a' mediante un fluorómetro de campo (10-005,
Turner Desings) calibrado por espectrofotometría. En las muestras de agua se midió posteriormente el fósforo total (PT) por digestión con ácido sulfúrico y nítrico (APHA, 1989).

\section{Estado trófico}

Para definir el estado trófico de una masa de agua y tener un dato comparativo con otros sistemas se utilizan índices del estado trófico calculados en base a diferentes parámetros. Un tipo de evaluación del estado trófico se basa en comparar los datos obtenidos experimentalmente con unos valores fijos propuestos para cada rango (Tabla 2). La OCDE (1982) utiliza la media anual de fósforo total (PT, mg. m-'), la media anual eufótica de clorofila 'a' (Chla, mg. $\mathrm{m}^{-3}$ ), el valor máximo anual de clorofila 'a' (Chla max, mg. m') y la profundidad media anual de visión del disco de Secchi (Sec, m).

Otro método para evaluar objetivamente el estado trófico es el índice de CARLSON (1974), que utiliza como variables los valores medios anuales de la profundidad de visión del disco de Secchi y de las concentraciones superficiales de PT y de clorofila 'a': 
TABLA 2. Clasificación trófica basada en límites fijos propuesta por la OCDE (1982). Ver texto.

TABLE 2. Trophic status by OECD (1982). See text.

\begin{tabular}{|c|c|c|c|c|}
\hline & PT ( $\left.\mathrm{mg} / \mathrm{m}^{3}\right)$ & Chla (mg / $\mathbf{m r}^{\mathbf{r}}$ ) & Chl max (mg / $\left./ \mathrm{ml}^{\prime}\right)$ & $\operatorname{Sec}(\mathbf{m})$ \\
\hline \multicolumn{5}{|l|}{ [1] } \\
\hline trófico & $\leq 4$ & $\leq 1$ & $\leq 2.5$ & $\geq 12$ \\
\hline Oliggorrófice & $\leq 10$ & $\leq 2,5$ & $\leq 8$ & $\geq 6$ \\
\hline Mesotrófico & $10-35$ & $2.5-8$ & $8-25$ & 6.3 \\
\hline Fintrífico & 35.100 & $8-25$ & 25.75 & $3-1.5$ \\
\hline Hijerenutrófico & $\geq 100$ & $\geq 25$ & $\geq 75$ & $\leq 1.5$ \\
\hline
\end{tabular}

TSI $=60-14,41 \ln$ Secchi

$\mathrm{TSI}=14,42 \ln \mathrm{PT}+4,14$

TSI $=9,81$ ln Chla $+30,6$

Este índice proporciona valores que pueden variar entre 0 (oligotrofia) y 100 (eutrofia), situándose en torno a 50 el límite con la mesotrofia.

\section{Modelos relativos al fósforo}

a) Coeficiente de retención, carga interna y liberación de fósforo del sedimento

El coeficiente de retención del fósforo $\left(R_{p}\right)$, introducido por DILLON \& RIGLER (1974) mide la proporción del fósforo que entra, que sedimenta de forma neta.

$$
\mathrm{R}_{\mathrm{p}}=1 \text { - }(\text { cantidad } \mathrm{P} \text { sale } / \text { cantidad } \mathrm{P} \text { entra })=\mathrm{R} \text { observado }
$$

Numerosos autores han revisado y comparado las diferentes fórmulas que existen para el cálculo del R $_{p}$ (NURNBERG, 1984; AHLGREN ef al. 1988; MARSDEN, 1989; FOY, 1992). En general, los modelos para el cálculo de $\mathrm{R}_{\mathrm{p}}$ tienden a sobrestimar la retención de fósforo, sobre todo los obtenidos para lagos óxicos cuando son aplicados a sistemas que desarrollan anoxia. Según NÜRNBERG (1984) los lagos con hipolimnion anóxico tienen un $\mathrm{R}_{\mathrm{P}}$ bajo e incluso negativo, debido a un aporte adicional de fósforo al sistema procedente de los sedimentoso carga interna de $\mathrm{P}\left(\mathrm{L}_{\mathrm{int}}\right)$. Puede suponer más del $91 \%$ de la entrada total de $\mathrm{P}$ al sistema y es la causa fundamental del retardo en la recuperación de sistemas eutróficos una vez que se reduce la carga externa $\left(\mathrm{L}_{\text {ext }}\right.$ ) (NURNBERG, 1984; NURNBERG \& PETERS, 1984).

Si consideramos un sistema como anóxico cuando presenta en su hipolimnion una concentración menor de $0,5 \mathrm{mg} 02.1^{1}$ durante al menos dos semanas y menos de siete meses
(NÜRNBERG, 1984), la diferencia entre el coeficiente de retención observado y el esperado $\left(R_{o b s}-R_{\text {esp }}\right)$ puede ser una estima de la carga interna $\left(L_{\text {int }}, \mathrm{mg} . \mathrm{m}^{2}\right.$ año $\left.{ }^{1}\right)$ :

$$
\begin{aligned}
& \mathrm{L}_{\text {int }}=-\mathrm{L}_{\text {ext }}\left(\mathrm{R}_{\text {obs }^{-}} \mathrm{R}_{\text {esp }}\right) \\
& \text { siendo } \mathrm{R} \text { esp }=15 /\left(18+\mathrm{q}_{\mathrm{S}}\right) \quad \text { (NÜRNBERG, 1984) } \\
& \mathrm{L}_{\text {ext }}=\text { carga externa de } \mathrm{P}\left(\mathrm{mg} . \mathrm{m}^{2} \text { año-1 }\right) \\
& \mathrm{q}_{\mathrm{s}}=\text { carga hidraúlica }\left(\mathrm{m} . \text { año }{ }^{1}\right)
\end{aligned}
$$

Si no disponemos del valor de $\mathrm{R}$ obs, existe otro método para el cálculo de la carga interna de $\mathrm{P}$ a partir del área de sedimento anóxico, de la duración de la anóxia y de la tasa de liberación de $\mathrm{P}$ del sedimento:

$\mathrm{L}_{\text {int }}=\mathrm{RR} . \mathrm{AF}$

(NÜRNBERG et al. 1986; NURNBERG, 1987 a)

$\mathrm{AF}=$ factor anóxico (días)

$\mathrm{RR}=$ tasa de liberación de $\mathrm{P}\left(\mathrm{mg} \cdot \mathrm{m}^{2}\right.$ día $\left.^{ }\right)$

$\mathrm{AF}=\sum$ (área de sedimento anóxico.días de anoxia) / área superficial del lago

La carga interna, $L_{\text {int }}$, varía de un año para otro debido a los cambios en la extensión de los sedimentos anóxicos y en la duración de la estratificación. Si calculamos según NÜRNBERG et al.(1986) el incremento de fósforo total hipolimnético in situ a partir de la diferencia entre el contenido en fósforo durante la estratificación y la cantidad previa epilimnética del verano -método 1-o a partir de la diferencia entre la cantidad máxima hipolimnética detectada y la cantidad presente justo antes del inicio de la anoxia - método 2obtendremos la Lint al dividir estos valores entre el área superficial del lago (NÜRNBERG, 1987 a).

b) Modelos empíricos para el cálculo del fósforo y niveles límite de aportación.

Existe un gran número de modelos empíricos que relacionan la aportación de fósforo a un lago o embalse $\left(\mathrm{L}_{\text {ext }}\right)$ con la concentración de fósforo resultante $\left(\mathrm{P}_{\mathrm{c}}\right)$. Deben tener en cuenta los efectos de la sedimentación y de la renovación hídrica, además de posibles aportes desde el sedimento.

Los modelos empíricos, obtenidos a partir de tratamientos estadísticos de datos de un gran número de sistemas, basan sus fórmulas en varias suposiciones, como son: la situación del sistema en estado estacionario, es decir, que al cabo de un tiempo, generalmente un año, la variación de la cantidad de $\mathrm{P}$ es nula y en que el sistema acuático se encuentre limitado por P. Su popularidad deriva de la simplicidad matemática y de los datos relativamente limitados que son necesarios para su 
aplicación, siendo de gran utilidad para el seguimiento de la eutrofización y la gestión de las masas de agua. Por el contrario, los modelos dinámicos han de ser calibrados para cada caso concreto y tienen un grado de precisión mayor (AHLGREN et al. 1988; MARSDEN, 1989; VAN HUET, 1992).

La OCDE (1982) mediante un estudio internacional sobre eutrofización desarrolló numerosos modelos empíricos tanto para lagos como para embalses basados en diferentes parámetros de calidad de aguas. VOLLENWEIDER y KEREKES (OCDE, 1982) prefieren referirse al término $P_{c}$ como "la concentración del tributario corregida por la renovación hidraúlica". Esta carga normalizada de P no tiene en cuenta los aportes difusos puntuales por escorrentia o precipitación atmosférica.

Una vez desarrollados los criterios de calidad del agua en base a unos parámetros indicadores del grado de eutrofia es interesante relacionar mediante los modelos empíricos, el aporte de nutrientes con la concentración esperada y, determinar de esta forma, cual sería la carga crítica del sistema que conduciría a la eutrofización.

Los modelos desarrollados por VOLLENWEIDER (1976) y por la OCDE (1982) permiten obtener una idea de cuál es la carga externa crítica a partir de la cual el sistema puede estar eutrofizándose.

NURNBERG (1991) introduce el término carga externa umbral de fósforo, que es aquella carga externa a la cual el flujo hacia abajo de fósforo procedente de fuentes externas se iguala al flujo hacia arriba procedente del hipolimnion. Comparando los valores de carga crítica con los observados realmente podemos señalar si el sistema se encuentra en estado de autodepuración $\left(\mathrm{L}_{\mathrm{ext}}<\mathrm{L}_{\mathrm{c}}\right)$ o si está acumulando fósforo y consiguientemente aumentando su grado de eutrofia $\left(L_{\text {ext }}>L_{c}\right)$. Según este concepto, un sistema que reciba una carga externa de fósforo importante acumulará el exceso en sus sedimentos, apartando temporalmente el fósforo de la circulación. Cuando el sistema alcanza una situación límite y ya no admite más fósforo en sus sedimentos, genera otros mecanismos para eliminarlo. La liberación de fósforo por los sedimentos anóxicos permite que se pierda parte del mismo por renovación hidraúlica y el sistema se autodepure. Si se trata de un embalse con toma hipolimnética, la recuperación trófica se verá favorecida al ser eliminado más rápidamente (NURNBERG, 1987 b; NURNBERG et al. 1987). A pesar de ello, la liberación del fósforo acumulado en los sistemas puede mantener la productividad del sistema durante largo tiempo (LARSEN et al. 1981; SONDERGAARD et al. 1993).

\section{RESULTADOS Y DISCUSION}

\section{Evaluación del estado trófico}

En base a la clasificación trófica de límites fijos propuesta por la OCDE (1982) y de acuerdo con nuestros datos (Tabla 3) podemos señalar que la media anual de PT y la media anual eufótica de Chla clasifican a los embalses de Cernadilla y

TABLA 3. Parámetros indicadores del estado trófico. Se señalan las medias geométricas $(\mathrm{X})$, los valores mínimos y máximos de las variables y el número de datos (n).

TABLE 3. Parameters used in the trophic state determination. Geometric mean $(\mathrm{X})$, minimun and maximun values and number of data (n) are shown.

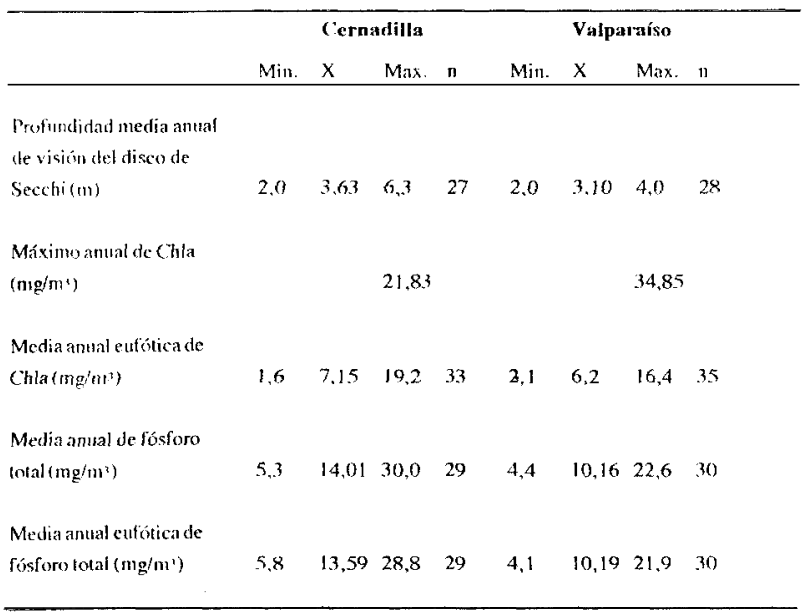

Valparaíso como mesotróficos, siendo Valparaíso el que más se acerca a la oligotrofia. Por otro lado, los valores máximos de clorofila 'a' señalan a Cernadilla como mesotrófico y a Valparaíso como eutrófico, mientras que según la profundidad media anual de visión del disco de Secchi ambos embalses serían mesotróficos, estando Valparaíso en el límite con la eutrofia.

Los valores del índice de CARLSON (1974) son inferiores a 50 , por lo que los embalses se clasifican como mesotróficos.

\section{Modelos relativos al fósforo \\ a) Coeficiente de retención, carga interna y liberación de fósforo del sedimento}

El coeficiente de retención de fósforo $\left(\mathrm{R}_{\mathrm{P}}\right)$ observado para Cernadilla y Valparaíso es de 0,20 y 0,13 , resoectivamente. Estos valores son relativamente bajos si se comparan con otros embalses españoles, como el de Sau (que tiene un $\mathrm{R}_{\mathrm{P}}$ superior a 0,50, según datos de VIDAL, en MARGALEF, 1983) y el de 
Aixola (Guipúzcoa) con un valor de 0,76 en 1992 (URRUTIA, 1994). La razón de estos valores tan bajos podría deberse a que la salida de agua hipolimnética en Cernadilla y Valparaíso facilita la pérdida de fósforo del sistema, ya que en el hipolininion es donde se acumula, tanto el fósforo que sedimenta, como el que se podría estar liberando desde los sedimentos.

Esta carga interna de fósforo $\left(\mathrm{L}_{\mathrm{int}}\right)$ se puede calcular de diferentes formas. Sin embargo, los modelos están pensados para lagos y asumen que la $\mathrm{L}_{\text {ext }}$ no cambia durante el periodo considerado, que no hay otras fuentes de $\mathrm{P}$ internas (macrófitos o zona litoral) y que el flujo es poco importante, por lo que son poco aplicables a los embalses. Así pues, la interpretación de los datos debe considerarse únicamente como orientativa y el incremento en PT hipolimnético ha de corregirse para las entradas y salidas. Dado que la toma para turbina y desagüe de fondo se encuentran en el hipolimnion, el cálculo de las salidas de $\mathrm{P}$ es sencillo. Sin embargo, por lo que respecta a las entradas de $\mathrm{P}$ por el tributario hemos considerado que, puesto que la mayor parte del fósforo está en forma particulada, aunque el agua circule a un nivel superior, el fósforo tenderá a sedimentar y por tanto engrosará la carga hipolimnética.

El modelo que aquí se propone, corregido para las entradas y salidas hipolimnéticas, tiene la siguiente forma (en mg. $\mathrm{m}^{-2}$ de área superficial del embalse y verano):

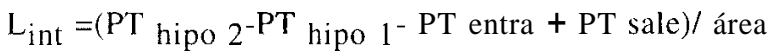
superficial embalse $=\mathrm{APT}_{\text {hipo }} /$ área superficial embalse

$\mathrm{PT}_{\text {hipo }}=$ cantidad de fósforo total $(\mathrm{kg})$ presente en el hipolimnion, al final del periodo considerado(2) y al principio(1)

A PT= incremento de fósforo total en el hipolimnion

Asumiendo que todo el incremento en fósforo total hipolimnético se debe a la liberación de los sedimentos anóxicos y que la magnitud de la tasa de liberación depende del estado trófico del sistema, los valores de carga interna observados (Tabla 4) califican a Cernadilla como eutrófico con $120.97 \mathrm{mg} . \mathrm{m}^{2}$ verano, y a Valparaíso como mesotrófico, con $30,92 \mathrm{mg} . \mathrm{m}^{2}$ verano (NÜRNBERG, 1988). Sin embargo, es muy probable que esos valores de tasa de liberación obtenidos a partir de determinaciones de la Lint in situ estén sobrestimados. El incremento en fósforo total hipolimnético se debe a una interacción compleja entre procesos físicos, químicos y biológicos, que suceden tanto en presencia de oxígeno como en anaerobiosis (BÖSTROM et al. 1988; MARSDEN, 1989; LOPEZ LASERAS, 1991). Pero en sistemas con producción elevada, como es nuestro caso, la sedimentación puede ser la principal causa del increinento en fósforo total del hipolimnion, como así lo apoya el hecho de

TABLA 4. Cálculo del factor anóxico (AF, días); del incremento de P’T hipolimnético (A PT hipo, Kg. valor medio obtenido a partir de los métodos $\mathbf{l}$ y 2); de la carga interna de PT determinada in situ ( $\mathrm{L}_{\mathrm{int}}$, , nig. $\mathrm{m}$ ' de áreá superficial del embalse y verano) y la rasa de liberación de PT del sedimento (RR, mg. $\mathrm{m}^{-1}$ de área de sedimento anóxico y días de anoxia) de los embalses de Cernadilla y Valparaíso durante el periodo de estratificación de 1991.

TABLE 4. Anoxic factor (AF, d), increased mass of TP hypolimnetic ( $\triangle \mathrm{P}^{\prime} T$ hipo. $\mathrm{Kg}$ ), internal TP load estimated (Lint, $\mathrm{mg} . \mathrm{m}^{-2}$ of reservoir surface area and summer), and TP release rate (RR, rng. $m$ ' of arioxic sediment surface and days of anoxia), during summer stratification in 1991 .

\begin{tabular}{|c|c|c|}
\hline & Cernadilla & Valparaíso \\
\hline AF (dias) & 8,4 & 5.0 \\
\hline$\left.\left.\Delta\right|^{x}\right|^{\prime} \operatorname{lipos}(\mathrm{Kg})$ & 774,33 & 376.10 \\
\hline Lint ng ['l /m: veranos] & 120,97 & 30.92 \\
\hline$R R(m g|y| m=$ dál & 14,40 & 6.2 \\
\hline
\end{tabular}

que no aumente la concentración de la fracción de fósforo reactivo total con la profundidad, sino únicaniente la total, que además está muy correlacionada con el seston orgánico y total y con el nitrógeno orgánico particulado (IBERDROLA, 1992; FRAILE, 1994). Esta relación entre el aumento del material orgánico y el fósforo total en el hipolimnion, está relacionada, también en otros embalses, con los cambios en la bioniasa fitoplanctónica durante la estratificación (PORCALOVA, 1990; RIERA et al. 1991).

En lagos oligotróficos que desarrollan anoxia, NÜRNBERG et al. (1986) calcularon la tasa de liberación de $\mathrm{P}$ de los sedimentos (RR) a partir de diferentes métodos, obteniendo un valor medio de 2,2 mg PT m² día'. Este fósforo liberado puede ser una fuente importante para el plancton epilimnético al final de verano y durante la mezcla otoñal ya que un porcentaje muy elevado del mismo, superior al $60 \%$ según NURNBERG et al. (1986), se encuentra en forma biodisponible. En Cernadilla y Valparaíso, los valores de RR son 14,40 y 6,2 mg PT $\mathrm{m}^{2}$ día ${ }^{1}$, respectivamente. Como era de esperar para estos sistemas mesoeutróficos, los valores obtenidos son superiores a los estimados para lagos oligotróficos.

b) Modelos empíricos para el cálculo del fósforo y niveles límite de aportación

En la Tabla 5 se señalan diferentes modelos para el cálculo de la concentración media de fósforo total $\left(\mathrm{Pc}, \mu \mathrm{g} \mathrm{I}^{\mathrm{s}}\right)$ a partir 
de los aportes de P, en los sistemas acuáticos. Los resultados que más se aproximan a nuestros datos experimentales se señalan

TABLA 5. Comparación entre la concentración media anual de PT ( $\mathrm{Pc}, \mu \mathrm{g} \mathbf{1}^{\text {') }}$ ) obtenida a partir de los datos de campo de 1991 y la estimada a partir de los modelos descritos en la bibliografía. Carga externa anual de PT $\left(L_{\text {ext }} \mathrm{mg}\right.$ año). coeficiente de retención observado ( $R$ obs $)$, coeficiente de retención esperado ( $R$ esp), carga hidráulica $\left(q_{s}\right.$, ni año $\left.{ }^{1}\right)$, tiempo de retención hidráulico (tw, años) y concentración media anual de PT en el agua de entrada (Pin, $\mu \mathrm{g} \mathrm{l}^{\prime}$ ). TABLE 5. Comparisons between predicted annual TP mean concentration based on empirical models $\left(\mathrm{Pc}, \mu \mathrm{g} \mathrm{I}^{-1}\right)$ and the TP mean calculated from field data in 1991. Exiernal TP load (Lext, mg m-' $\mathrm{yr}^{-1}$ ), observed phosphorus retention $\left(\mathbf{R}_{\text {obs }}\right)$, predicted phosphoriis retention $\left(\mathrm{R}_{\mathrm{esp}}\right)$, areal water load $\left(\mathrm{q}_{\mathrm{s}}, \mathrm{m} \mathrm{yr}^{-1}\right)$, water residence time ( $\mathrm{tw}, \mathrm{yr})$ and $\mathrm{TP}$ inflow $\left(\mathrm{P}_{\mathrm{in}}, \mu \mathrm{g} \mathrm{l}^{\prime}\right)$.

\begin{tabular}{|c|c|c|}
\hline Modeto & Cernadilla & Valparaíso \\
\hline \multicolumn{3}{|l|}{$P_{k}=1$ exl(1 Rohs $) / q^{5}$} \\
\hline (DHILON \& RIGLER. 1974) & 11.16 & $* 9.90$ \\
\hline \multicolumn{3}{|l|}{$\Gamma_{c}=(1, c \times 1 / q s) .1 /(1+v t w)$} \\
\hline (VOILENWEIDER, 1976) & 8.55 & 6.67 \\
\hline \multicolumn{3}{|l|}{$P c=1.551 \operatorname{Pin} /(1+\sqrt{ }(w))^{0.82}$} \\
\hline (OCLEE. 1982) Fómulula general & 12.38 & 7.32 \\
\hline \multicolumn{3}{|l|}{$\mathrm{P}_{\mathrm{c}}=1.02\left(\mathrm{Pin} /(1+\sqrt{ }(\mathrm{w}))^{0.88}\right.$} \\
\hline (OCDE. 1982) Fórmula embalses & 9,48 & 5.69 \\
\hline \multicolumn{3}{|l|}{$\mathrm{Pc}_{\mathrm{c}}=(1$ ext $\left./ \mathrm{q} s) \cdot 1 \mathrm{Resp}\right)+(\mathrm{I} \mathrm{int} / \mathrm{q} \mathrm{s})$} \\
\hline (NURBERG, 1984) & $* 14,43$ & $* 8,80$ \\
\hline \multicolumn{3}{|l|}{$P_{c}=(1.118$. Pin $) /(1+\sqrt{ }(w) 1.135$} \\
\hline (FOY. 1992) & $* 13,19$ & 6.91 \\
\hline Pcobservado $(\mu \mathrm{g} / 1)$ & 14.01 & 10,16 \\
\hline
\end{tabular}

con un asterisco, siendo el desarrollado por NÜRNBERG (1984), el que mejor se ajusta para los dos embalses. Esta autora mostró que la capacidad de predicción de los modelos publicados era a menudo pobre cuando se comparaban con los valores observados en lagos anóxicos, ya que se ignoraba la carga interna de fósforo, que puede ser muy importante en estos sistemas. Según FOY (1992) las diferencias pueden deberse a que en la elaboración de los modelos no se incluyeron lagos con tiempos de residencia bajos, inferiores a un año, a que se produzca una importante liberación de fósforo del sedimento en condiciones anóxicas, o a que parte del fósforo hipolimnético derive del plancton que sedimenta.

La carga externa de $\mathrm{P}$ oscila en los países desarrollados entre 0,1 y $1 \mathrm{~g} \mathrm{P} \mathrm{m}^{-2}$ año', siendo retenida por los sedimentos, entre el 5 y el $60 \%$. En el caso del embalse de Sau, la carga
TABLA 6. Comparación entre la carga externa anual de PT ( $\mathrm{L}_{\text {ext. }} \mathrm{mg} \mathrm{m}$ ' año '), según los modelos propuestos por varios autores. Concentración media anual de PT en el agua de entrada (Pin, $\mu \mathrm{g} \mathrm{l}^{+}$), volumen de descarga anual (Vol in, $\mathrm{m}^{\prime}$ año'), área superficial del sistema $\left(a, m^{\prime}\right)$, carga hidráulica $\left(q_{k}, m\right.$ año-'). tiempo de retención hidráulico (tw, años), profundidad media ( $z$, metros), carga interna de PT $\left(\mathrm{L}_{\mathrm{int}}, \mathrm{mg} \mathrm{m}^{-2}\right.$ año $\left.{ }^{1}\right)$, coeficiente de retención de fósforo esperado $\left(\mathrm{R}_{\mathrm{esp}}\right)$, área del hipolimnion (Ahipo, $\mathbf{m}^{2}$ ) y área de sedimento anóxico (A anox, $\mathrm{m}^{\prime}$ ).

TABLE 6. Comparison between external TP load $\left(\mathrm{L}_{\text {ext }}, \mathrm{mg} \mathrm{m}^{\prime} \mathrm{yr}^{-1}\right)$ and critical TP load $\left(L_{c}, m^{\prime} m^{\prime} y^{\prime}\right)$ based on several autors. TP inflow (Pin. $\mu \mathrm{g}^{1}$ ), water inflow (Vol in, $\left.\mathrm{m}^{\prime} \mathrm{yr}^{-1}\right)$, reservoir surface area $\left(\mathrm{A}, \mathrm{m}\right.$ ?), water load $\left(\mathrm{q}_{\mathrm{s}}, \mathrm{m}^{\prime} \mathrm{yr}^{-1}\right)$, water residence time (tw, ry), mean deapth $(\mathrm{z}, \mathrm{m})$, internnl TP load (Lint, $\mathbf{m g} / \mathbf{m}^{\text {: }}$ ry) predicted $P$ retention $\left(R_{\text {esp }}\right)$, hypolimnetic area $\left(a\right.$ hipo, $\left.m^{2}\right)$ and sedinient area overlain by anoxic water (A anox, $\left.\mathrm{m}^{\prime}\right)$.

\begin{tabular}{|c|c|c|}
\hline & Cernadilla & Valparaíso \\
\hline L_ext= Piul $\operatorname{Vol}$ in $/ \mathrm{A}\left(\mathrm{mg} / \mathrm{m}^{2}\right.$ año $)$ & 650,42 & 283,21 \\
\hline \multicolumn{3}{|l|}{$1 . c=10 ., \mathrm{ss}(1+\sqrt{ }(w)$} \\
\hline (O) CDE. 1982) & 761.05 & 424.72 \\
\hline \multicolumn{3}{|l|}{$L c=17\left(z /\lfloor x)^{0,6} . z^{0.4}\right.$} \\
\hline (VOLLLENWLIIDISR. 1976) & 549,40 & $320.5+1$ \\
\hline \multicolumn{3}{|l|}{ L.c-1/ int / Resp), (A hipo / A arox) } \\
\hline (NURBERG, 1991) & 718,48 & 270,69 \\
\hline
\end{tabular}

externa de fósforo total obtenida por VIDAL (1976) es niuy superior (más de 30 g P m:' año.') siendo el coeficiente de retención cercano a 0,50. TOJA et al., (1992) obtienen valores inferiores de carga externa: $0,36 \mathrm{~g} \mathrm{P} \mathrm{m}^{-2}$ año ${ }^{1}$ para el embalse de Aracena y 1,22 y $5,11 \mathrm{~g} \mathrm{P} \mathrm{m}^{-2}$ año $^{-1}$ para La Minilla y El Gergal, respectivamente. Los embalses de Cernadilla y Valparaíso, que se situan en una cuenca poco humanizada, poseen una carga externa de 650,42 y $283,21 \mathrm{mg} \mathrm{m}^{-2}$ año ', respectivamente, que disminuye en Valparaíso, debido al efecto regulador del embalse anterior. Sin embargo, la carga externa que reciban realmente será superior a la que se compara en la Tabla 6, puesto que la calculada corresponde únicamente al aporte del tributario.

Excepto para el modelo de la OCDE, según el cual ambos embalses reciben una carga externa inferior a la crítica, los demás modelos (Tabla 6) indican que Cernadilla podría estar acelerando su eutrofización, mientras que Valparaíso recibe una carga externa similar o ligeramente inferior a la crítica, por lo que estaría mejorando su estado trófico. Sin embargo, la eutrofización parece el destino de los embalses trás la recuperación inicial una vez mineralizada la materia orgánica de su cubeta (KOUSSOURIS et al., 1991; KILIKIDIS et al., 1992). No obstante, en el caso de Cernadilla que presentaba condiciones oligotróficas antes de la construcción de Valparaíso (MARGALEF et al., 1976; CASADO et al., 1989). el empeoramiento en su trofía se debe a la gestión. La pérdida de volumen embalsado concentra los nutrientes y aumenta la 
relación entre la profundidad de la zona fótica y la profundidad media. MARIAZZl et al., (1992) constataron el fenómeno contrario, es decir, una mejora importante en la calidad del agua de un embalse al producirse el cambio en la gestión. Este cambio condujo a un aumento de la profundidad media, minimizando las fluctuaciones de nivel, lo que supuso una disminución de la relación profundidad fótica/profundidad media, dejando una proporción mayor de la carga nutritiva fuera de la zona fótica.

Antes de la construcción de Valparaíso, Cernadilla presentaba características oligotróficas. El cambio fundamental que ha experimentado posteriormente ha consistido en la disminución de su volumen por la pérdida del hipolimnion durante el periodo de la estratificación. Esto condiciona un aumento de su estado trófico, paliado en parte por la salida hipolimnética, que favorece la eliminación de las sustancias acumuladas. En este caso, el deterioro de la calidad del agua durante la estratificación, no se debe a un incremento de la carga externa, como es frecuente en otros embalses (BAYNE et al., 1990; KOUSSOURIS et al., 1991; KILIKIDIS et al., 1992), sino a cambios morfométricos ocasionados por la gestión. La disminución de volumen favorece la desestratificación temprana en Cernadilla, lo que puede tener un efecto ambiguo sobre la calidad del agua. Por un lado favorece la difusión de oxígeno hacia las zonas profundas, que ya no están aisladas por una termoclina efectiva, pero por otro, al contar con una gran extensión de sedimento epilimnético, puede poner a disposición de los productores primarios abundantes nutrientes, aumentando su productividad (NÜRNBERG, 1987 b; JAMES \& BARKO, 1991).

Valparaíso, tres años después de su llenado, ha mejorado la calidad de las aguas embalsadas. La mineralización de la materia contenida en la cubeta trás su inundación, supuso una liberación brusca de nutrientes, responsable de los síntomas de eutrofia detectados por LIMNOS S.A. (1989). Sin embargo, la toma hipolimnética favorece la eliminación de los nutrientes acumulados en el hipolimnion, provenientes tanto de la liberación del sedimento, como de la descomposición de la materia orgánica alóctona o autóctona. Las salidas hipolimnéticas favorecen, en general, la recuperación de los sistemas eutróficos (NURNBERG, 1987 b; NURNBERG et al., 1987), evitando la meromixis biogénica que, en casos de eutrofia elevada, podría incluso dificultar la mezcla total (BOWLING \& TYLER, 1990).

\section{AGRADECIMIENTOS}

Este artículo ha sido posible gracias a la financiación por parte de IBERDROLA S.A. del proyecto de investigación:
"Gestión medioambiental de embalses. Los embalses del río Tera", incluido en el proyecto PIE 121.43-EVALUACIÓN DE CAUDALES ECOLÓGICOS. También agradecemos a José Carlos Vega Ureta su colaboración y seguimiento de las campañas quincenales de muestreo.

\section{BIBLIOGRAFÍA}

AHLGREN, I., FRISK, T. \& L. KAMP-NIELSEN, 1988. Empirical and theoretical models of phosphorus loading, retention and concentration vs. lake trophic state. Hydrobiologia, 170: 285-303.

AMERICAN PUBLIC HEALTH ASSOCIATION, 1989. Standard methods for the examination of Water, Sewage, and Wastewater. 17th Ed. American Public Health Association, Washington, D.C. 1550 pp.

BAYNE, D.R., SEESOCK, W.C., WEBBER, C.E. \& J.A. McGUIRE, 1990. Cultural eutrophication of West Point Lake-a 10 year study. Hydrobiologiu, 199: 143-156.

BENGOECHEA, C. 1991. Valparaíso dam-Tera river firts filling experiences. International Commission on Large Darns, 64: 67-83.

BÖSTROM, B., ANDERSEN, J.M., FLEISCHER, S. \& M. JANSSON, 1988. Exchange of phosphorus across the sediment-water interface. Hydrobiologia, 170: 229-244.

BOWLING, L.C. \& P.A. TYLER, 1990. Chemical stratification and partial meromixis in reservoirs in Tasmania. Hydrobiologia, 194: 67-83.

CARLSON, R.E. 1974. A trophic state index for lakes. Limnol. Oceanogr., 23: 361-369.

CASADO, C., GARCIA DE JALON, D., DEL OLMO, C.M., BARCELO, E. \& F. MENES, 1989. The effect of an irrigation and hydroelectric reservoii- on its downstream communities. Regulated Rivers: Res. \& Manag., 4: 275-284.

DILLON, P.J. \& F.H. RIGLER, 1974. The chlorophyll-phosphorus relationship in lakes. Lirnnol. Oceanogr., 19: 767-773.

FOY, R.H. 1992. A phosphorus loading model for Northern Irish lakes.Wat. Res., 26: 633-638.

FRAILE, H. 1994. Limnología comparada del sistema de embalses del río Tera (Zamora). Tesis Doctoral. 232 pp.

IBERDROLA. 1992. Gestión medioambiental de embalses. Informe equipo embales Julio, UPV, Bilbao.

JAMES, W.F.\& J.W. BARKO, 1991. Estimation of phosphorus exchange between littoral and pelagic zones during nighttime convective circulation. Limnol. Ocearzogr., 36(1): 179-187.

KILIKIDIS, S., KAMARIANOS, A., KARAMANLIS, X. \& S. DELLIS, 1992. Water quality and thophic status evaluation of the polyphyto reservoir, N. Greece.Toxicological and 
Environmental Chemistry, 36: 169-179.

KOUSSOURIS, T.S., DIAPOULIS, A.C. \& G.D. PHOTIS, 1991. Evaluating the trophic status of a shallow polluted lake, lake Ioannina. Greece.Toxicological and Environmental Chemistry, 31-32: 303-313.

LARSEN, D.P., SCHULTS, D.W. \& K.W. MALUEG, 1981. Summer internal phosphorus supplies in Shagawa Lake, Minnesota. Limnol. Oceanogr., 26: 740-753.

LIMNOS S. A. 1989. Estudio de la calidad del agua de los embalses del sistema Cernadilla-Valparaíso y su relación con las poblaciones piscícolas del río Tera. Barcelona. Informe realizado para Iberdrola S.A. Bilbao.

LÓPEZ LASERAS, P. 1991. Sedimentary phosphorus dynamics in epicontinental systems: a literature review. Homage to Ramón Margalef; or, Why there is such pleasure in studying nature. (ROS, J. D. \& N. PRAT, eds). Oecol, aquat., 10: 113-125.

LÓPEZ, P. \& J.A. MORGUI, 1993. Factors influencing fractional phosphorus composition in sediments of Spanish rcscrvoirs. Hydrobiologia, 253: 73-82.

MARGALEF, R., PLANAS, D., ARMENGOL, J., VIDAL, A., PRAT, N., GUISET, A., TOJA, J. \& M. ESTRADA, 1976. Limnología de los embalses españoles.Serv. Publ. Direc. Gral. Obras Hidraúlicas. M.O.P. Madrid. 422 pp.

MARGALEF, R. 1983. Limmnología. Ed. Omega, Barcelona. $1010 \mathrm{pp}$.

MARSDEN, M.W. 1989. Lake restoration by reducing external phosphorus loading: the influence of sediment phosphorus release. Freshwater Biol., 21: 139-162.

MARIAZZI. A.A., DONADELLI, J.L., ARENAS, P., DI SIERVI, M.A. \& C. BONETTO, 1992. Impact of a nuclear power plant on water quality of Embalse del Río Tercero Reservoir, (Córdoba, Argentina).Hydrobiologia, 246: 129-140.

NURNBERG, G.K. 1984. The prediction of internal phosphorus load in lakes with anoxic hypolimnia. Limnol. Oceanogr., 29(1): 111-124.

NURNBERG, G.K. 1987 a. A comparison of internal phosphorus loads in lakes with anoxic hypolimnia: Laboratory incubation versus in situ hypolimnetic phosphorus accumulation.Limnol. Oceanogr., 32(5): 1160-1164.

NURNBERG, G.K. 1987 b. Hypolimnetic withdrawal as lake restoration technique. Journal of Environmental Engineering, 113(5): 1006-1017.

NURNBERG, G.K. 1988. Prediction of phosphorus release rates from total and reductant-soluble phosphorus in anoxic lake sediments. Can. J. Fish. Aquat. Sci., 45(1): 453-462.

NURNBERG, G.K. 1991. Phosphorus from internal sources in the Laurentian Great Lakes, and the concepts of threshold external load. J. Great Lakes Res., 17(1): 132-140.
NURNBERG, G.K., DILLON, P.J. \& D.J. McQUEEN, 1986. Internal phosphorus load in an oligotrophic Precambrian shield lake with an anoxic hypolimnion. Can. J. Fish. Aquat. Sci., 43(3): 574-580.

NURNBERG, G.K., HARTLEY, R. \& E. DAVIS, 1987. Hypolimnetic withdrawal in two North American lakes with anoxic phosphorus release from the sediment.Wat. Res., 21(8): 923-928.

NURNBERG, G.K. \& R.H. PETERS, 1984.The importance of internal phosphorus load to the eutrophication of lakes with anoxic hypolimnia. Verh. Internat. Verein. Limnol., 22: 190-194.

OCDE, 1982. Eutrophisation des eaux. Métodes de surveillance, d'evaluation et de lutte. Paris. 164 pp.

PORCALOVA, P. 1990. Phosphorus losses fron the epilimnion in Rímov reservoir. Int. Revue ges. Hydrobiol., 75(3): 273-279.

POZO, J., ORIVE, E., FRAILE, H. \& A. BASAGUREN (en prensa). Effects of the Cernadilla-Valparaíso reservoir system on the river Tera. Regulated Rivers: Res. \& Manag.

RIERA, J. L., MARTI, E. \& J . A. MORGUI, 1991. Changes in the trophic state of the Spanish reservoirs during the last sixteen years.Verh. Internat. Verein Limnol., 24: 1367-1370.

RIERA, J. L., JAUME, D., DE MANUEL, J., MORGUI, J. A. \& J. ARMENGOL, 1992. Patterns or variation in the limnology of Spanish reservoirs: A regional study. Limrzética, 8: 111-123.

SONDERGAARD, M., KRISTENSEN, P. \& E. JEPPESEN, 1993. Eight years of internal phosphorus loading and changes in the sediment phosphorus profile of lake Sobygaard, Denrnark. Hydrobiologia, 253: 345-356.

TOJA, J., BASANTA, A. \& R. FERNANDEZ ALES, 1992. Factors controlling algal biomass in the complex of water supply reservoirs of Seville (Spain). Limnética, 8: 267-277.

URRUTIA, J.I. 1994. Estudio limnológico de los embalses de Aixola, Urkulu, Barrendiola y Lareo, 1988-1992. Gipuzkoa. Tesis doctoral.

VAN HUET, H.J.W.J. 1992. Phosphorus eutrophication in the SW Frisian lake district 1. Monitoring and assessment of a dynamic mass balance model. Hydrobiologia, 233: 259-270.

VEGA, J.C., de HOYOS, C. \& J.J. ALDASORO, 1992. The Sanabria lake. The largest natural freshwater in Spain. Limnética, 8: 49-57.

VIDAL, A. 1976. Eutrofización del embalse de Sau en el transcurso de sus primeros años (1963-1972). Confed. Hidrogr. del Pirineo Oriental, secc $I^{o}$ de Explotación Aguas del Ter, Cardedeu, Barcelona. 43 pp.

WOLLENWEIDER, R.A. 1976. Advances in defining critical loading levels for phosphorus in lake eutrophication. Mem. Ist. Ital. Idrobiol., 33: 53-84. 